\title{
A new variety of Hosta (Liliaceae): Hosta clausa var. geumgangensis M. Kim \& H. Jo
}

\author{
Hyun Jo and Muyeol Kim*
}

Department of Biological Sciences, Chonbuk National University, Jeonju 54896, Korea

(Received 9 September 2016; Revised 19 September 2016; Accepted 23 September 2016)

\section{비비추속(백합과)의 신변종: \\ 금강비비추(Hosta clausa var. geumgangensis M. Kim \& H. Jo) \\ 조현 · 김무열* \\ 전북대학교 자연과학대학 생명과학과}

\begin{abstract}
A new variety, Hosta clausa Nakai var. geumgangensis M. Kim \& H. Jo, was found in Geumgang River in Korea. This variety shares several characteristics (rhizomes with underground stolon, smooth scape and purple-colored anthers) with the reólated varieties H. clausa Nakai var. clausa and H. clausa var. normalis F. Maekawa. However, H. clausa var. normalis has open flowers, capsules with seeds, purple-spotted petioles, long pistils, and long narrow perianth tubes, whereas this new variety has open flowers, seedless capsules, green-colored petioles, short pistils, and short narrow perianth tubes. Hosta clausa Nakai var. clausa has closed flowers and seedless capsules.
\end{abstract}

Keywords: Hosta clausa var. geumgangensis, plantain lilies, Lilliaceae, endemic taxa

적 요: 전북 진안군 금강변에서 비비추속의 신변종인 금강비비추(Hosta clausa Nakai var. geumgangensis M. $\mathrm{Kim} \& \mathrm{H} . \mathrm{Jo}$ )를 새로이 발견하여 기재하였다. 금강비비추는 근경에 기는 줄기가 있고, 종주선이 화경에 없 으며, 약은 자색이라는 점에서 비비추 H. clausa var. normalis나 참비비추 H. clausa var. clausa와 유사하다. 그러나 금강비비추는 개방화이고, 종자 결실을 하지 않으며, 엽병이 녹색이며, 암술과 수술의 길이가 비슷하고, 화피 협통부는 길이가 짧다. 반면에, 비비추는 개방화이고, 종자 결실을 하며, 엽병은 자색 반점이 있으며, 암술 은 수술보다 길고, 화피 협통부는 길이가 길다. 또한 참비비추는 꽃의 화피가 전혀 열리지 않는 폐쇄화이고 종자를 맺지 못한다.

주요어: 금강비비추, 비비추류, 백합과, 고유분류군

Genus Hosta Tratt. (Lilliaceae) consists of approximately 22 to 25 species (Jones, 1989). It is endemic genus in Eastern Asia (Maekawa, 1940).

Plants in this genus have the following characteristics: leafless scape, bisexual flower, perianth tube, and a capsule
(Chung, 2007). Seven species of Hosta are distributed in Korea, including $H$. plantaginea (Lam.) Asch. that has been introduced from China (Chung and Chung. 1988; Chung and Kim. 1991; Chung, 2007).

Hosta clausa Nakai is comprised of two varieties. H. clausa

*Author for correspondence: mykim@jbnu.ac.kr

Open Access http://e-kjpt.org, (C) 2016 the Korean Society of Plant Taxonomists. This is an open-access article distributed under the terms of the Creative Commons Attribution Non-Commercial License (http://creativecommons.org/licenses/by-nc/4.0) which permits unrestricted non-commercial use, distribution, and reproduction in any medium, provided the original work is properly cited. 
Table 1. Comparison of some morphological characters among Hosta clausa var. geumgangensis, H. clausa var. normalis, and H. clausa var. clausa. Ranges and means in parenthesis are provided for quantitive charcters.

\begin{tabular}{|c|c|c|c|}
\hline Characters & H. clausa var. geumgangensis & H. clausa var. normalis & H. clausa var. clausa \\
\hline Leaf blade length (cm) & $5.9(12.6) 20.5$ & $4.4(13.2) 21.2$ & $8.0(11.5) 20.0$ \\
\hline Leaf blade width (cm) & $1.4(3.4) 5.0$ & $1.6(4.5) 8.8$ & $1.1(1.8) 2.9$ \\
\hline Petiole length $(\mathrm{cm})$ & 4.7 (11.6) 22.0 & $2.5(18.0) 30.0$ & $6.5(13.0) 24.0$ \\
\hline Petiole abaxial color & Green & Purple-spotted green & Green \\
\hline Inflorescence length $(\mathrm{cm})$ & $58(74) 103$ & $21(50) 76$ & $34(61) 78$ \\
\hline Flower number & $14.0(24.5) 45.0$ & $5.0(19.4) 35.0$ & $11.0(25.8) 58.0$ \\
\hline Pedicel color & Purple & Whitish purple to purple & Purple \\
\hline Pedicel length (mm) & $5(7) 10$ & $4(8) 14$ & $6(9) 14$ \\
\hline Perianth length $(\mathrm{cm})$ & $2.8(3.7) 3.9$ & $3.8(4.8) 5.8$ & $3.3(3.5) 3.8$ \\
\hline Perianth width $(\mathrm{cm})$ & $2.0(2.7) 3.5$ & $3.0(3.6) 4.1$ & $0.80(0.82) 0.92$ \\
\hline Narrow tube length $(\mathrm{cm})$ & $1.0(1.1) 1.2$ & $1.6(1.8) 2.2$ & $1.3(1.4) 1.5$ \\
\hline Wide tube length $(\mathrm{cm})$ & $1.2(1.3) 1.4$ & $1.5(1.8) 2.2$ & $1.0(1.2) 1.4$ \\
\hline Perianth lobe & Open & Open & Closed \\
\hline Perianth lobe length $(\mathrm{cm})$ & $1.4(1.5) 1.7$ & $1.3(1.6) 1.8$ & $0.9(1.0) 1.1$ \\
\hline Perianth lobe width $(\mathrm{cm})$ & $0.7(0.8) 1.0$ & $0.7(1.0) 1.3$ & $0.4(0.5) 0.6$ \\
\hline Pistil length $(\mathrm{cm})$ & $4.2(4.5) 4.8$ & $5.5(6.3) 7.6$ & $3.3(3.5) 3.8$ \\
\hline Stamen length $(\mathrm{cm})$ & $4.0(4.2) 4.5$ & $4.3(5.3) 6.3$ & . \\
\hline Distance between pistil and stamen $(\mathrm{cm})$ & $0.1(0.3) 0.5$ & $0.5(0.9) 1.6$ & . \\
\hline Capsule & Seedless & With seeds & Seedless \\
\hline Distribution & Southern Korea & Northern and central Korea & Central Korea \\
\hline
\end{tabular}

var. clausa has closed flowers and seedless capsule and $H$. clausa var. normalis F. Maekawa has open flowers and a capsule with seeds (Nakai, 1930; Maekawa, 1937, 1940; Schmid, 1991; Jo, 2016). H. clausa var. ensata (F. Maekawa) W. G. Schmid is a synonym for $H$. clausa var. normalis (Jo, 2016).

A new taxon of Hosta from Geumgang River, Jinan-eup, Jinan-gun, Jeonbuk Province, Korea was discovered. The new variety shares several characters with its related variety, $H$. clausa var. normalis F. Maekawa. However, it can be distinguished from its related species by having seedless capsule (versus a capsule with seeds), green-colored petioles (versus green with purple spot), short pistils (versus long pistils), and short narrow part of perianth tube (versus long narrow part of perianth tube) (Table 1, Appendix 1). We named this new taxon as Hosta clausa Nakai var. geumgangensis M. Kim \& H. Jo.

\section{Taxonomic Treatment}

1. Hosta clausa Nakai var. geumgangensis M. Kim \& H. Jo, var. nov. (Figs. 1-3).
TYPE: KOREA. Jeonbuk Province: Jinan-gun, Jinan-eup, along Geumgang River, 24 Aug 2015, H. Jo JF82402 (holotype, JNU; isotypes, JNU, KH, KB).

Korean name: Geum-gang-bi-bi-chu 금강비비추.

Diagnosis: Hosta clausa Nakai var. geumgangensis can be distinguished from its related taxa by having seedless capsule (versus a capsule with seeds), green-colored petioles (versus green with purple spot), short pistils (versus long pistils), and short narrow part of perianth tube (versus long narrow part of perianth tube) (Fig. 4).

Herbs perennial, glabrous. Rhizome stout, usually with underground stolon. Leaves 7-11, basal; petiole green, 4.7$22.0 \mathrm{~cm}$ long, winged at least in distal part; blades 5.9$20.5 \mathrm{~cm} \times 1.4-5.0 \mathrm{~cm}$, oblong to narrow elliptical, glabrous, apex acute, veins 3-6 pairs. Inflorescence spike-like raceme, elongate, 58-103 $\mathrm{cm} \times 2.5-6.0 \mathrm{~mm}, 14-45$-flowered; scape smooth; bracts pale yellow or green at flowering, ovate to broad ovate or elliptical, 6-9 $\mathrm{mm} \times 4-6 \mathrm{~mm}$, membranous; pedicel purple, 5-10 $\mathrm{mm}$ long. Flowers opening during the day, odorless; perianth purple, bell-shaped, $2.8-3.9 \mathrm{~cm} \times 2.0$ 

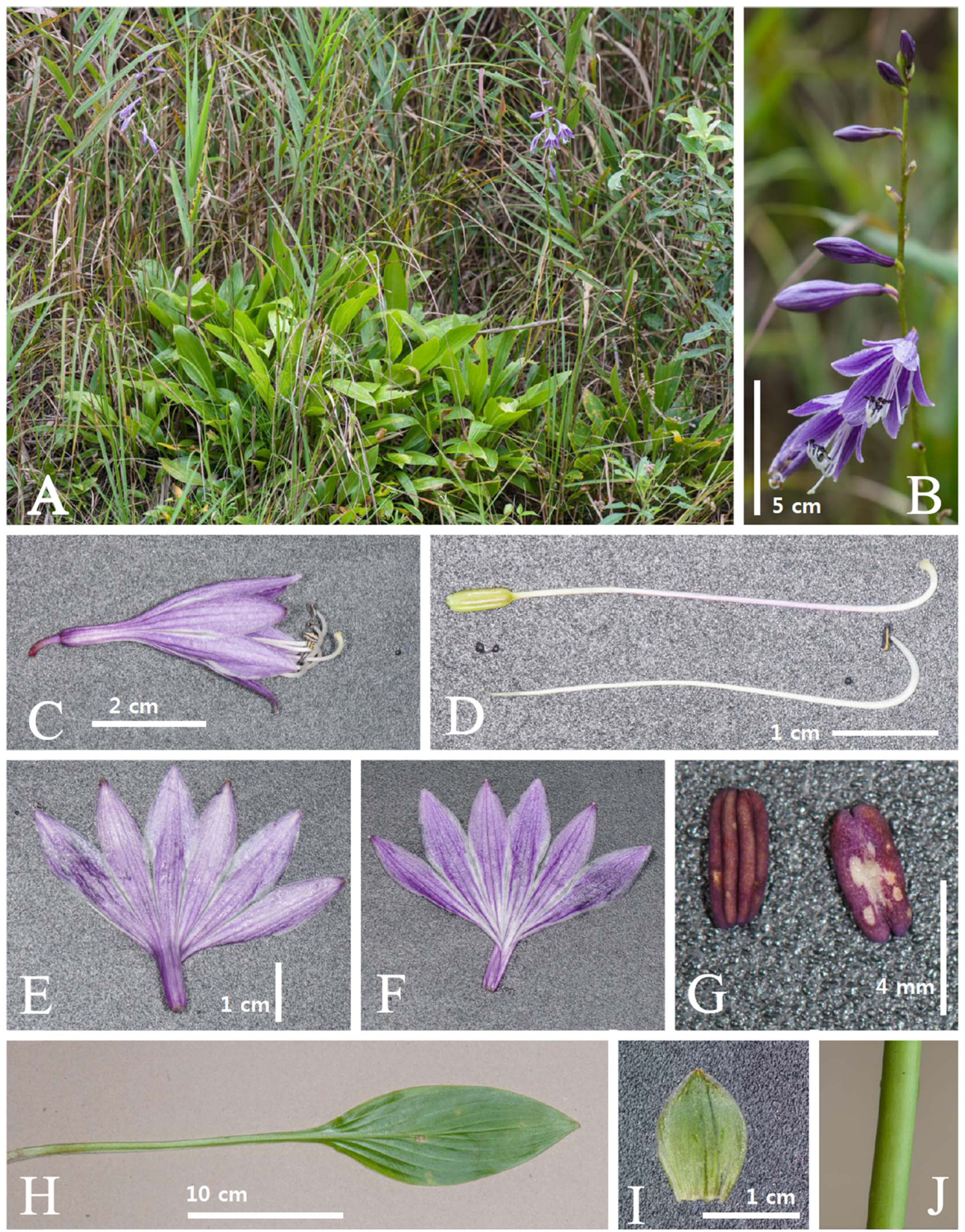

Fig. 1. A-J. Photographs of Hosta clausa Nakai var. geumgangensis M. Kim \& H. Jo at type locality. A. Habit. B. Inflorescence. C. Perianth. D. Pistil \& Stamen. E. Perianth (outer side). F. Perianth (inner side). G. Anther color. H. Leaf. I. Bract. J. Scape. 


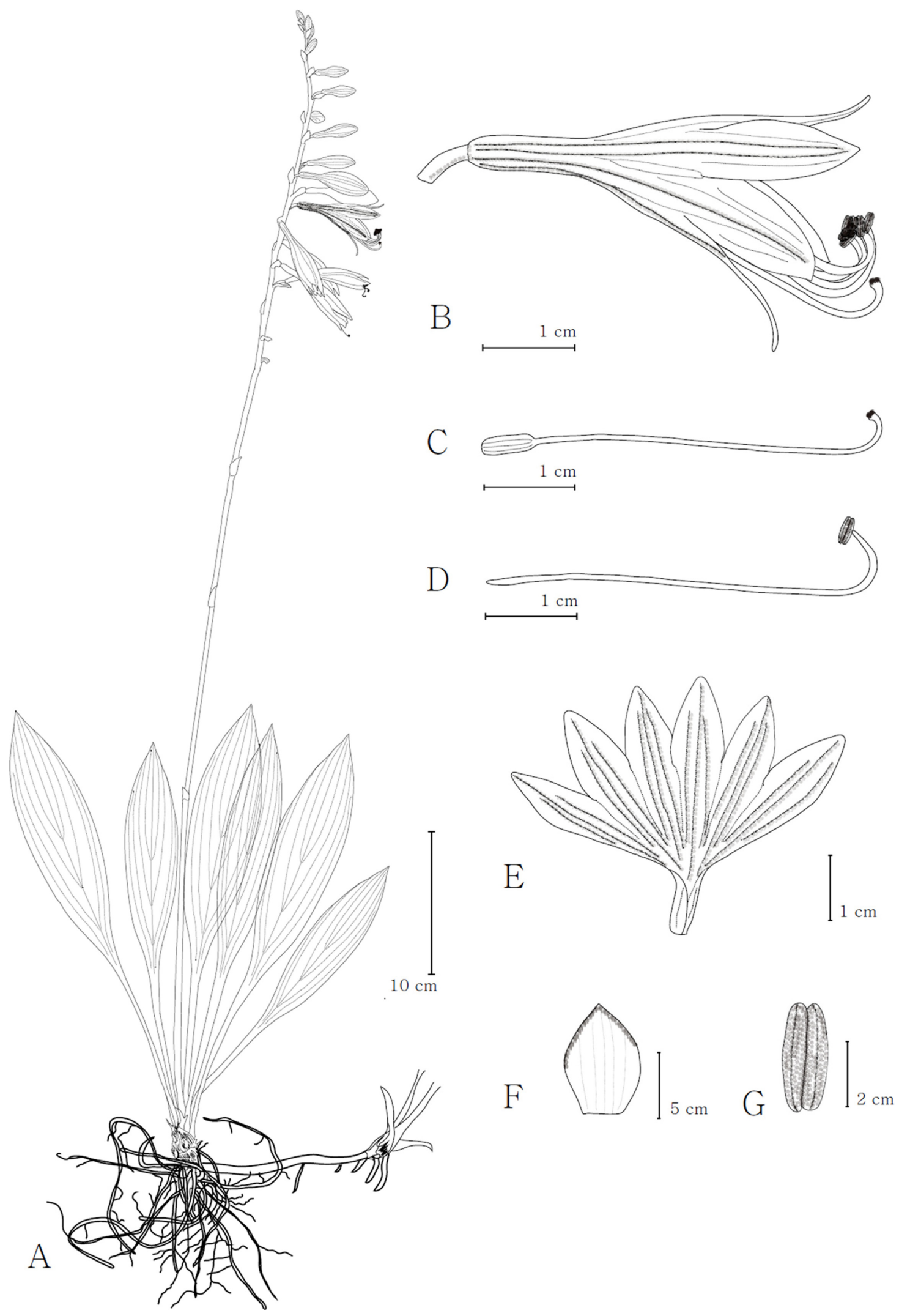

Fig. 2. Hosta clausa Nakai var. geumgangensis M. Kim \& H. Jo. A. Adult plant with flowers. B. Flower. C. Pistil. D. Stamen. E. Perianth (inner side). F. Bract. G. Anther. 


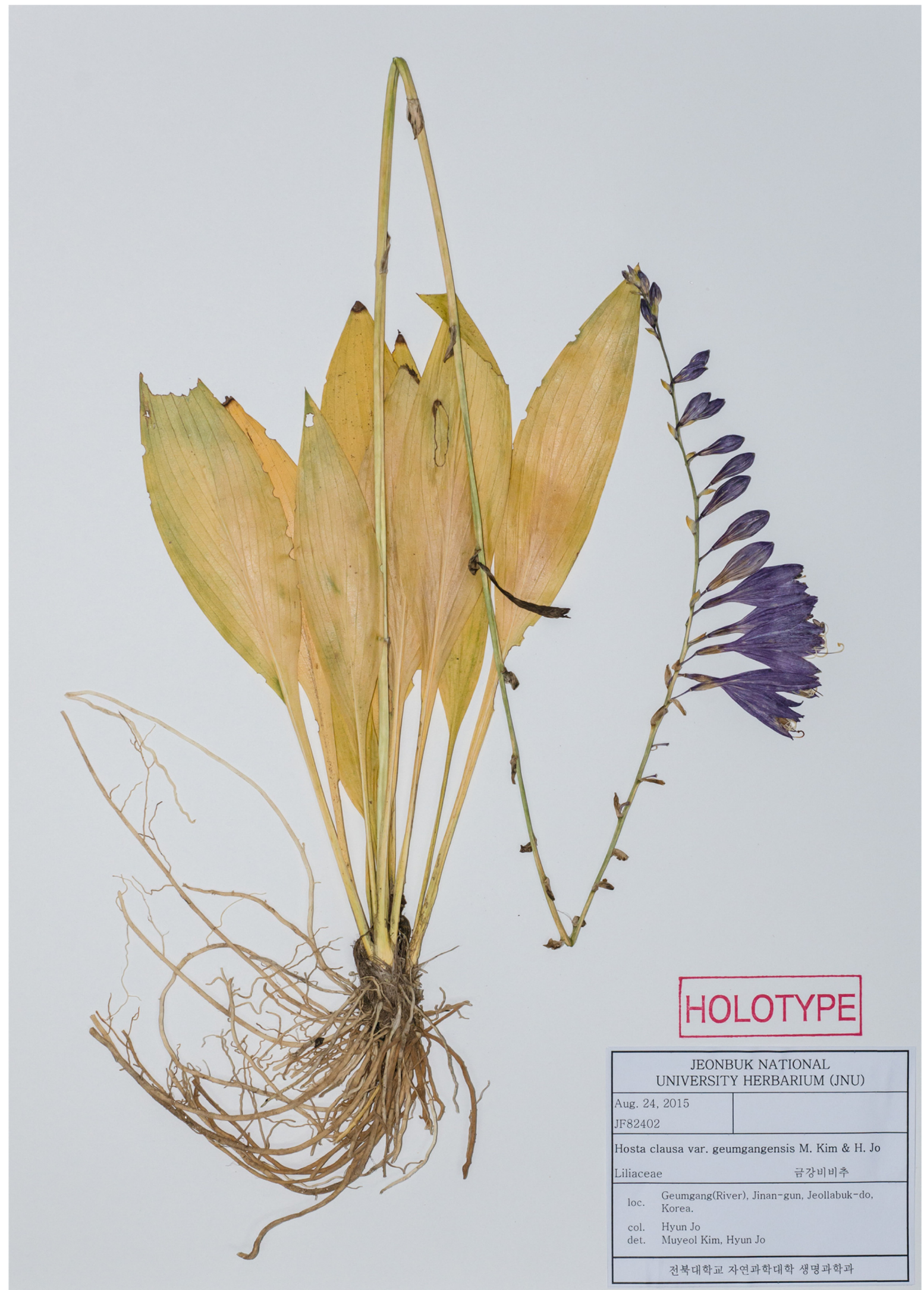

Fig. 3. Holotype of Hosta clausa Nakai var. geumgangensis M. Kim \& H. Jo. 

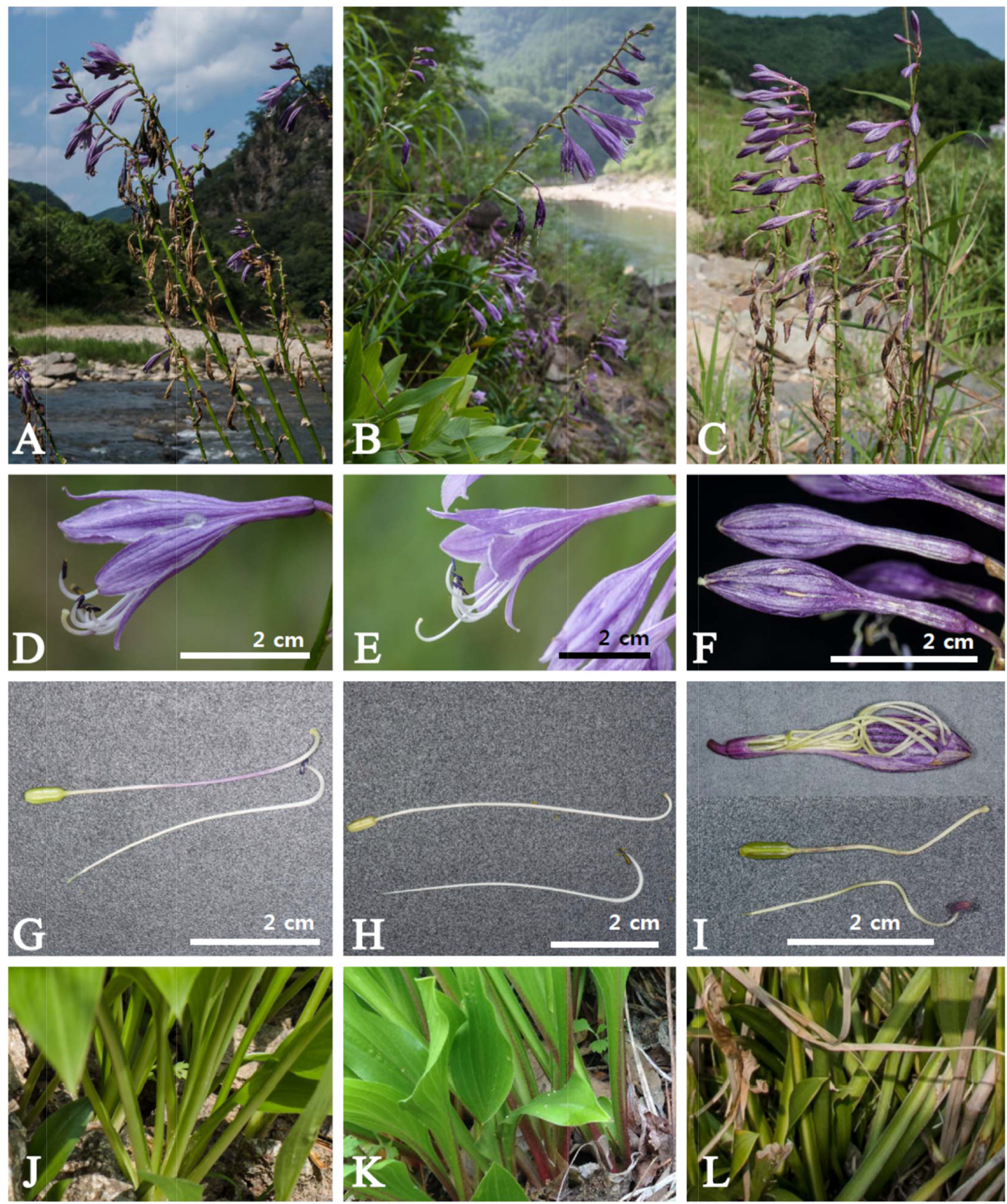

Fig. 4. Photographs of morphological comparisons among Hosta clausa varieties in Korea. A, D, G, J. H. clausa var. geumgangensis. B, E, H, K. H. clausa var. normalis. C, F, I, L. H. clausa var. clausa. A-C. Inflorescence. D-F. Perianth. G-I. Pistil and stamen. J-L. Petiole abaxial color.

$3.5 \mathrm{~cm}$; narrow parts of the perianth grooved, $1.0-1.2 \mathrm{~cm}$ long; wide parts of the perianth $1.2-1.4 \mathrm{~cm}$ long; lobes 6 ,
1.4-1.7 cm × 0.7-1.0 cm, opened. Stamens 6, equal, 4.0$4.5 \mathrm{~cm}$ long, slightly exerted; filament white; anther purple, 
$3.4-4.1 \mathrm{~mm} \times 1.6-1.8 \mathrm{~mm}$. Pistil $4.2-4.8 \mathrm{~cm}$ long, style whitish purple; distance between pistil and stamen 0.1$0.5 \mathrm{~cm}$. Capsule seedless.

Flowering: Early Aug to mid Sep.

Distribution: Southern Korea (Jinan-gun, Jeollabuk-do).

Etymology: The infraspecific epithet was derived from its type locality (Geumgang River, Jinan-eup, Jinan-gun, Jeonbuk Province, Korea).

Habitat: Large population of Hosta clausa var. geumgangensis is found at sandy soil area of Geumgang river. This new variety grows at river edges dominated by Phragmites communis and Phragmites japonica. This species also grows with other herbaceous species dominated by Lythrum anceps, Oenothera biennis, Acer tataricum subsp. ginnala, and Humulus japonicus.

Additional specimens examined (paratypes): Korea, Jeonbuk Province, Jinan-gun, Jinan-eup, along Geumgang River, 29 Aug 2015, H. Jo, JF82901-JF82903; 26 Jul 2016, H. Jo, JG72601-JG72605 (JNU); 2 Aug 2016, H. Jo, JG80201JG80204 (JNU); 22 Aug 2016, H. Jo, JG82201 (JNU).

\section{Key to Hosta clausa var. geumgangensis and its related taxa}

1. Flower more than $10 \mathrm{~cm}$ long, flagrant, opening at night H. plantaginea

1. Flower less than $7 \mathrm{~cm}$ long, odorless, opening during the day.

2. Scape ridged.

3. Racemes congested, head-like ……. H. capitata

3. Racemes elongate, spike-like ……….... H. minor

2. Scape smooth.

4. Flower funnel-shape. Stamens 3 short and 3 long

4. Flower bell-shape. Stamens 6 equal.

5. Rhizome without underground stolon. Anthers yellow-color ……........................... H. jonesii

5. Rhizome with underground stolon. Anthers purple-color.

6. Flowers closed …... H. clausa var. clausa

6. Flowers opened.

7. Petiole green with purple spot. Perianth $3.8-5.8 \mathrm{~cm}$ long; narrow parts of the perianth tube $1.6-2.2 \mathrm{~cm}$ long. Capsule with seeds ….. H. clausa var. normalis
7. Petiole green without purple spot. Perianth 2.8-3.9 cm long; narrow parts of the perianth tube $1.0-1.2 \mathrm{~cm}$ long. Capsule seedless

............ H. clausa var. geumgangensis

Using the IUCN Red List Categories and Criteria (IUCN, 2011), Hosta clausa var. geumgangensis M. Kim \& H. Jo is categorized as Endangered (EN) species because only one thousand to one thousand five hundreds organisms have been found at sandy soil area of Geumgang River, Korea.

\section{Acknowledgments}

We are deeply indebted to Hyun-Sook Choi for her kind help.

\section{Literature Cited}

Chung, M. G. and J. W. Kim. 1991. The genus Hosta Tratt. (Liliaceae) in Korea. SIDA 14: 411-420.

Chung, Y. C. and Y. H. Chung. 1988. A taxonomic study of the genus Hosta in Korea. Korean Journal of Plant Taxonomy 18: 161-172. (in Korean)

Chung, Y. C. 2007. Hosta. In The Genera of Vascular Plants of Korea. Park, C. W. (ed.), Academy Publishing Co., Seoul. Pp. 1304-1306.

IUCN. 2011. Guidelines for Using the IUCN Red List Categories and Criteria. Version 3.1. Retrieved Oct. 7, 2015, from http:// www.iucnredlist.org.

Jo, H. 2016. A taxonomic study of the genus Hosta in Korea. Ms thesis, Chonbuk National University, Jeonju. Pp. 46-48, 5460. (in Korean)

Jones, S. B. 1989. Hosta yingeri (Liliaceae/Funkiaceae): a new species from Korea. Annals of the Missouri Botanical Garden 76: 602-604.

Maekawa, F. 1937. Divisiones et plantae novae generis Hostae. Journal of Japanese Botany 13: 898-905.

Maekawa, F. 1940. The genus Hosta. Journal of the Faculty of Science, Imperial University of Tokyo, Section 3 Botany 5: 317 425.

Nakai, T. 1930. Notulae ad Plantas Japoniae et Koreae 38. Botanical Magazine Tokyo 44: 27.

Schmid, W. G. 1991. The genus Hosta: Giboshi Zoku. Timber Press, Portland, OR. Pp. 43-44. 


\section{Appendix 1. Examined specimens of Hosta clausa varieties in Korea.}

\section{Hosta clausa Nakai var. normalis F. Maekawa}

KOREA. Gangwon-do: Cheolwon-gun, 23 Aug 2015, H. Jo, JF82301, JF82302, JF82303 (JNU); 29 Jul 2015, M. Kim, MKH07291, MKH07292 (JNU); Gangneung-si 28 Sep 2012, K. S. Lee et al., 628 (KH); Goseong-gun, 22 Jul 1999, Y. M. Lee, S-2324 (KH); Jeongseon-gun, 24 Aug 2016, H. Jo, JG82407, JG82408, JG82409 (JNU); Hongcheon-gun, 6 Sep 2016, H. Jo, JG90601, JG90602, JG90603, JG90604, JG90605, JG90606, JG90607, JG90608 (JNU). Gyeonggi-do: Pocheon-si, 23 Aug 2015, H. Jo, JF82304, JF82305, JF82306, JF82307, JF82308, JF82309, JF82310 (JNU); Yeoncheon-gun, 23 Aug $2015, H$. Jo, JF82311, JF82312 (JNU).

Hosta clausa Nakai var. clausa

KOREA. Chungcheongbuk-do: Goesan-gun, 23 Aug 2016, H. Jo, JG82302, JG82303, JG82304, JG82305, JG82306, JG82307, JG82308, JG82309, JG82310, JG82311, JG82312 (JNU). 